\title{
Análise da mobilidade toracoabdominal de idosos com doença de parkinson, submetidos ao treinamento funcional, bicicleta estacionária e exergame: um ensaio clínico randomizado
}

\author{
Analysis of the thoracoabdominal mobility of elderly parkinson's disease that have \\ done stationary bicycle, functional training and exergaming: a randomized controlled \\ trial
}

Gabriel Pereira Duarte ${ }^{1}$, Nildo Manoel da Silva Ribeiro ${ }^{2 *}$, Daniel Dominguez Ferraz ${ }^{2}$

${ }^{1}$ Mestrando em Processos Interativos dos Órgãos e Sistemas do Instituto de Ciências da Saúde ICS/UFBA.; ${ }^{2}$ Professor do Departamento de Fisioterapia, da Universidade Federal da Bahia.

\begin{abstract}
Resumo
Introdução: idosos com doença de Parkinson (DP) apresentam mobilidade toracoabdominal reduzida, o que os predispõe a complicações respiratórias, como a pneumonia aspirativa. Objetivo: avaliar a mobilidade toracoabdominal em idosos com doença de Parkinson, antes e após realizarem treino funcional, bicicleta estacionária e exergame. Metodologia: 58 idosos foram randomizados em três grupos: um que realizou treino funcional (GF) $n=18$, outro que fez bicicleta estacionária (GB) $n=20$ e o terceiro que realizou o exergame (GE) n=20. A mobilidade foi mensurada pela cirtometria, por avaliador cego, em três níveis: axilar, xifoideano e umbilical, antes e após as intervenções. Resultados: não foram encontradas diferenças estatisticamente significativas no coeficiente de amplitude nos níveis axilar e xifoide e umbilical nos três grupos. No nível umbilical, entretanto, houve um discreto acréscimo, comparando antes e após as intervenções GF de 2,1(4,52) cm para $3,1(4,27) \mathrm{cm}$; GB de $1,1(3,16) \mathrm{cm}$ para $1,7(4,68) \mathrm{cm}$ e GE de $0,5(3,29) \mathrm{cm}$ para $1,0(3,68) \mathrm{cm}$. Conclusão: os três programas de exercícios propostos não foram eficazes na melhora da mobilidade torácica no nível axilar e xifoide e parecem provocar uma pequena melhora na mobilidade abdominal, no contexto respiratório.

Palavras-chave: Idoso. Exercício. Doença de Parkinson. Fisioterapia.
\end{abstract}

\begin{abstract}
Introduction: elderly with Parkinson's disease (PD), have reduced thoracoabdominal mobility, which predisposes to respiratory complications such as aspiration pneumonia. Objective: to assess thoracoabdominal mobility in elderly with PD before and after performing functional training, stationary bike and exergaming. Methodology: fifty eight (58) elderly people were randomized into three groups: functional training (GF) $n=18$, stationary bike (GB) $n=20$ and exergaming (GE) $n=20$. Mobility was measured by cirtometry, by blind assessor, in three levels: axillary, xiphoid and umbilical; before and after the interventions. Results: no statistically significant differences were found in the amplitude coefficient at axillary and xiphoid and umbilical levels in the three groups. At the umbilical level, however, there was a slight increase comparing before and after GF interventions from 2.1(4.52) cm to $3.1(4.27) \mathrm{cm}$; $G B$ of $1.1(3.16) \mathrm{cm}$ to $1.7(4.68) \mathrm{cm}$ and GE of $0.5(3.29) \mathrm{cm}$ to $1.0(3.68) \mathrm{cm}$. Conclusion: the three exercise programs proposed were not effective in improving axillary and xiphoid thoracic mobility and appear to cause a slight improvement in abdominal mobility in the respiratory context.
\end{abstract}

Keywords: Elderly. Exercise. Parkinson's disease. Physiotherapy.

\section{INTRODUÇÃO}

A doença de Parkinson (DP) é considerada uma enfermidade neurodegenerativa que se caracteriza como um distúrbio dos neurônios dopaminérgicos da substância negra do mesencéfalo ${ }^{1,2}$. Dentro do seu quadro sintomático, destacam-se as alterações posturais, temores, rigidez e bradicinesia ${ }^{3}$. Apesar das repercussões motoras serem as mais evidentes, o sistema respiratório também é alvo da DP, desde o seu início. Dada a exuberância dos

Correspondente/Corresponding:*Nildo Manoel da Silva Ribeiro - Universidade Federal da Bahia, Instituto de Ciências da Saúde - End: Av. Reitor Miguel Calmon, s/n, Vale do Canela CEP 40170010 - Salvador — BA - Tel.: (71) 98194-1458 - E-mail: Nildoribeiro67@gmail.com sintomas motores, por vezes a função respiratória, que também está comprometida, acaba sendo negligenciada.

A postura em flexão do tronco, a mobilidade reduzida da caixa torácica (rigidez) e a degeneração osteomioarticular, figuram entre os principais sintomas respiratórios na $\mathrm{DP}^{4}$. A diminuição dos volumes e capacidades pulmonares, além da fraqueza muscular respiratória, surgem em consequência dessas alterações ${ }^{4}$. Considerada como a principal causa de óbito na DP, a pneumonia aspirativa está diretamente relacionada à incapacidade de mobilizar secreções e a um bom funcionamento do mecanismo da tosse, que, por sua vez, acaba sendo comprometido devido às alterações da função respiratória 5 .

O tratamento usual da DP envolve aspectos farma- 
cológicos e não farmacológicos, sendo que dentre estes a Fisioterapia se destaca como um componente fundamental no processo de reabilitação neurológica ${ }^{6}$. Entre os recursos terapêuticos utilizados estão os exercícios de força e condicionamento muscular, treino de transferências, posturas, equilíbrio, além do condicionamento cardiorrespiratório ${ }^{7}$. 0 exergame, por meio da realidade virtual, tem surgido como uma alternativa diante das opções mais tradicionais de tratamento. Atenção, participação e motivação do paciente são requeridas para integrar o movimento corporal com os sistemas dos jogos de computador utilizados pelo exergame ${ }^{8}$.

A cirtometria, também conhecida como perimetria toracoabdominal, é uma medida da circunferência do tórax e abdômen, nos momentos de inspiração e expiração máxima; para tal, é necessária apenas uma fita métrica, que fornece um parâmetro de mobilidade relacionada a respiração?.

Assim sendo, este estudo teve como objetivo avaliar a mobilidade toracoabdominal, através da cirtometria, de idosos com DP, submetidos a três diferentes modalidades de tratamento: o treino funcional, a bicicleta estacionária e o exergame, antes e após essas intervenções.

\section{METODOLOGIA}

Trata-se de um estudo tipo ensaio clínico randomizado, duplo cego, composto por 58 idosos com DP, de ambos os gêneros, em estágio leve a moderado da doença, randomizados em três grupos: treino funcional (GF), bicicleta estacionária (GB) e exergame (GE).

A pesquisa atendeu as diretrizes definidas na Resolução no 466, do Conselho Nacional de Saúde de 2012, sendo aprovada no Comitê de Ética em Pesquisa do Instituto de Ciências da Saúde/UFBA, através do Parecer no 1.016.971.

Foram definidos como critérios de inclusão: estar em uso regular da medicação para a DP e em período "on", ter idade igual ou maior que 60 anos, apresentar estágio 2, 2,5 ou 3 segundo a Classificação de Hoehn e Yahr modificada ${ }^{10}$, ler e assinar o Termo de Consentimento Livre e Esclarecido (TCLE). Foram considerados critérios de exclusão: apresentar doenças neurodegenerativas, excluindo-se a DP, demências, doenças ostearticulares e musculares que impossibilitassem a prática de atividade física, doenças crônicas não controladas, doenças cardiovasculares instáveis, uso de álcool e outras substâncias tóxicas, contraindicações para a realização de exercício físico segundo os critérios do Colégio Americano de Medicina do Esporte ${ }^{11}$, ter praticado programa de exercício físico nos últimos 6 meses ou ter participado de treinamentos regulares de resistência (por exemplo, 2-3 vezes por semana) nos 12 meses anteriores ao início do estudo.

As sessões realizadas no GF, GB e GE tiveram uma duração de 8 semanas, com uma frequência de 3 sessões de 50 minutos por semana. Cada sessão, em todos os grupos, foi composta por: 10 minutos de alongamentos,
5 minutos de exercícios calistênicos, 30 minutos de intervenção e 5 minutos de exercícios respiratórios para desaquecimento ${ }^{7,12}$.

Um mesmo fisioterapeuta realizou o tratamento com o Xbox360 e sensor KinectTM e outro o treino funcional e em bicicleta estacionária, durante as 24 sessões.

Durante as intervenções, os pacientes foram monitorizados quanto à frequência cardíaca $(\mathrm{FC})$, saturação periférica de oxigênio (Spo2) e escala de Borg ${ }^{13}$, além da observação de sinais, como palidez, cianose e diaforese súbita; foram orientados a informar aos pesquisadores quaisquer sintomas, como lipotimia, dispneia, dor, palpitações, ainda se o paciente viesse a apresentar alteração da $F C \geq 40 \mathrm{bpm}$ e da PAS $\geq 20 \mathrm{mmHg}$ do basal, relato de Borg $\geq 17, \mathrm{SpO} 2<90 \%$ a atividade era interrompida ${ }^{12}$.

O GF, composto por 18 indivíduos, foi submetido ao treino funcional. Este treinamento consistiu em 30 minutos de exercícios em um circuito constituído por 10 atividades, com duração de 3 minutos cada. Entre as atividades funcionais, encontravam-se as seguintes estações: 1) marcha com obstáculos; 2) subir e descer escada e rampa; 3) levantar e sentar; 4) marcha de lado; 5) exercício de equilíbrio em plataforma proprioceptiva; 6) atividades com bola; 7) exercício de descer e subir estepes; 8) exercícios de ponta de pé; 9) exercícios de alcance; 10) marcha com caneleiras. A evolução da intensidade das atividades descritas foi realizada através de resistência progressiva, com o uso de halteres, caneleiras e bandas elásticas, visando alcançar uma percepção de cansaço individual correspondente a 15 pontos da Escala de Borg ${ }^{14}$.

Os participantes do GB, composto por 20 indivíduos, realizaram um treinamento aeróbico em bicicleta estacionária, com duração de 30 minutos. A intensidade do treino na primeira semana foi de $50 \%$ da frequência cardíaca (FC) e aumentou progressivamente até alcançar $75 \%$ da $\mathrm{FC}$ na oitava semana. A frequência cardíaca de treino foi calculada pela equação: (FCmáx - FCrep) x intensidade + Fcrep. A FCmáx (frequência cardíaca máxima) foi estimada pela equação (220 - idade) ${ }^{16}$ e a FCrep (frequência cardíaca de repouso) foi aferida após 10 minutos de repouso em sedestração ${ }^{16,17}$

O tratamento do GE, composto por 20 indivíduos, foi realizado por 30 minutos de exercícios com jogos do videogame Xbox360, com sensor KinectTM. Foram utilizados 2 a 3 jogos por sessão e a intensidade do exercício foi determinada pelo grau de dificuldade do jogo para alcançar uma percepção de cansaço individual, correspondente a 15 pontos da Escala de Borg.

A amplitude toracoabdominal foi mensurada em centímetros por meio da cirtometria, tendo como referência três níveis horizontais: axilar, xifoide e abdominal. No nível axilar, a fita métrica foi posicionada na dobra axilar; no xifoide foi colocada no apêndice xifoide; e no abdominal, foi utilizada a distância média entre o apêndice xifoide e a cicatriz umbilical. A medida foi realizada com os indivíduos na posição de pé, em cada um dos níveis foram solicitadas três inspirações e expirações máximas, por meio do 
comando verbal do avaliador; o coeficiente de amplitude foi obtido pela diferença dos valores da inspiração e expiração máximas, sendo considerada como medida válida o maior valor obtido entre as mensurações em cada nível ${ }^{9}$.

No que se refere à dinâmica das avaliações, os participantes foram avaliados no período da manhã e se encontravam em fase on das medicações para o tratamento da DP.

Sobre o tratamento estatístico dos dados, utilizou-se o teste de Kolmogorov-Smirnov para avaliar a distribuição das variáveis estudadas. Calcularam-se média e desvio padrão como medidas de tendência central e dispersão para as variáveis quantitativas e as frequências absoluta e relativa para as qualitativas coletadas. Ao realizar os procedimentos para a escolha do teste estatístico adequado à comparação ou correlação, optou-se por utilizar o teste T para comparar as variáveis dependentes e o Teste ANOVA para variáveis independentes. $O$ teste qui-quadrado foi utilizado para comparar as variáveis categóricas entre os grupos.

\section{RESULTADOS}

Um total de 69 idosos foi inicialmente selecionado para participar do estudo; destes, 5 desistiram; foram, então, randomizados para os três grupos: 64 idosos, 21 para o GF, mas 3 não conseguiram realizar os procedimentos de avaliação da função pulmonar; assim, restaram 18 para a análise, 22 para o GB, sendo que 2 não conseguiram realizar os procedimentos de avaliação da função pulmonar, restando 20 para a análise; e 22 para o GE, dos quais 2 não conseguiram realizar os procedimentos de avaliação da função pulmonar, restando 20 para a análise (Figura1).

Figura 1 - Diagrama do fluxo de entrada e perdas das fases de um estudo randomizado com três grupos de intervenção em Salvador, Brasil, 2019.

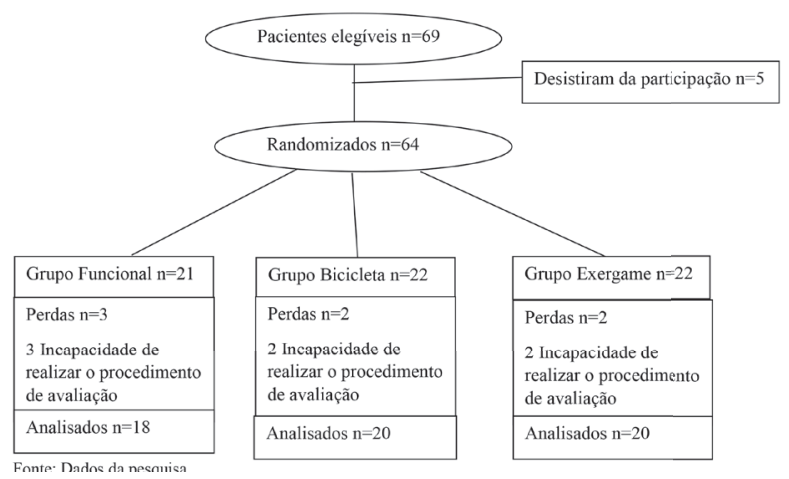

Fonte: Dados da pesquisa.

Permaneceram para análise um total de 58 idosos, 36 (62\%) do sexo masculino e 22 (38\%) do sexo feminino, estando a caracterização dos três grupos descritas no Quadro 1.
Quadro 1- Características demográficas e clinicas dos idosos com $D P$

\begin{tabular}{|lccccccc|}
\hline Variáveis & GF $(\mathbf{n}=\mathbf{1 8})$ & GB $(\mathbf{n = 2 0})$ & \multicolumn{3}{c|}{ GE $(\mathbf{n = 2 0})$} \\
\hline & Média (DP) & Média (DP) & Média (DP) & $\mathbf{p}$ \\
\hline Sexo (feminino) & 14 & $(4)$ & 12 & $(8)$ & 10 & $(10)$ & 0,206 \\
Idade (anos) & 70,56 & $(04,61)$ & 68,00 & $(05,41)$ & 67,30 & $(03,09)$ & 0,074 \\
Escolaridade (anos) & 08,72 & $(03,69)$ & 09,20 & $(03,51)$ & 07,65 & $(03,68)$ & 0,392 \\
IMC (Kg/m $)$ & 26,53 & $(03,84)$ & 23,89 & $(04,20)$ & 26,61 & $(05,82)$ & 0,131 \\
UPDRS & 27,06 & $(12,27)$ & 28,65 & $(12,86)$ & 32,30 & $(15,48)$ & 0,479 \\
Tempo de doença & 06,22 & $(04,98)$ & 06,65 & $(03,66)$ & 05,75 & $(03,68)$ & 0,789 \\
Hoehn and Yahr & 02,61 & $(00,36)$ & 02,50 & $(00,39)$ & 02,42 & $(00,33)$ & 0,302 \\
\hline
\end{tabular}

Fonte: Dados da pesquisa.

Legenda: $n$ : número de participantes; DP: desvio padrão; IMC: índice de massa corporal; UPDRS: Escala Unificada de Avaliação da doença de Parkinson.

De acordo com os dados do quadro, os grupos se apresentaram de forma homogênea quanto a idade, índice de massa corpórea (IMC), tempo de doença e a classificação de Hoehn e Yahr, com exceção do grupo funcional GF que tem a maioria dos seus participantes do sexo masculino.

No Quadro 2, estão apresentados os resultados da comparação da cirtometria torácica medida antes e depois das intervenções em cada grupo; os valores estão expressos em centímetros, resultado do coeficiente de amplitude (CA) obtido pela diferença de valores medidos entre uma inspiração e expiração máximas.

Quadro 2 - Diferença da cirtometria torácica antes e após intervenção por grupo

\begin{tabular}{|c|c|c|c|c|c|c|}
\hline Variáveis & $\begin{array}{l}\text { GF }(n=18) \\
\text { Média (DP) }\end{array}$ & p & $\begin{array}{l}\text { GB }(n=20) \\
\text { Média (DP) }\end{array}$ & p & $\begin{array}{l}\text { GE }(n=20) \\
\text { Média (DP) }\end{array}$ & $p$ \\
\hline \multirow[t]{2}{*}{ Axilar } & $6,1 \quad(2,03)$ & 0,248 & $6,0 \quad(1,99)$ & & $6,3 \quad(2,15)$ & \\
\hline & $6,6 \quad(1,33)$ & & $6,6 \quad(1,35)$ & 0,101 & $6,3 \quad(1,32)$ & 0,953 \\
\hline \multirow[t]{2}{*}{ Xifoidiana } & $5,7 \quad(2,26)$ & 0,704 & $5,4 \quad(2,56)$ & & $5,6 \quad(1,78)$ & \\
\hline & $5,5 \quad(1,09)$ & & $5,7 \quad(1,96)$ & 0,588 & $5,7 \quad(1,69)$ & 0,695 \\
\hline \multirow[t]{2}{*}{ Abdominal } & $2,1 \quad(4,52)$ & 0,503 & $1,1 \quad(3,16)$ & & $0,5 \quad(3,29)$ & \\
\hline & $3,1 \quad(4,27)$ & & $1,7 \quad(4,68)$ & 0,592 & $1,0 \quad(3,68)$ & 0,369 \\
\hline
\end{tabular}

Fonte: Dados da pesquisa.

Legenda: GF: grupo funcional; GB: grupo bicicleta; GE: grupo exergame; $D P$ : desvio padrão, os valores estão expressos em centímetros.

Conforme demonstrado no Quadro 2, não foram encontradas diferenças estatisticamente significantes antes e após as intervenções, nos três níveis de medição nos três grupos.

\section{DISCUSSÃO}

Apesar dos resultados não apresentarem a chamada "significância estatística" e não representarem nada além da chance do acaso explicar ou não os resultados obti$\operatorname{dos}^{18}$, quando observamos os valores do coeficiente de amplitude no nível abdominal, houve uma diferença de no mínimo 0,5 centímetros $(\mathrm{cm})$, nos três grupos, sendo 
que no GF essa diferença chegou a $1 \mathrm{~cm}$ na diferença da média obtida antes e depois das intervenções.

Cardoso e Pereira ${ }^{19}$, em estudo que comparou a mobilidade de indivíduos com DP e não parkinsonianos (NP), demostraram a diminuição dessa mobilidade de forma importante, sendo encontrada uma diferença de $1,8 \mathrm{~cm}$ no coeficiente de amplitude dos indivíduos com DP e de 4,3 nos NP, medida essa realizada no nível xifoidiano.

Tasca, Schuster e Alvarenga ${ }^{20}$ encontram uma variação média de 3,3 cm entre a inspiração e a expiração máxima medida ao nível do apêndice xifoide, sendo que os participantes não realizaram nenhum tipo de intervenção.

Já Ramos et al. ${ }^{4}$ citam em seu estudo que $70 \%$ da sua amostra apresentam a expansibilidade torácica pouco móvel ou rígida, não fornecendo os dados medidos ou de que forma se realizou essa classificação.

Em uma revisão da literatura realizada por Costa et al. ${ }^{21}$ sobre as alterações funcionais respiratórias na DP, dos 17 artigos selecionadas para integrarem a revisão apenas 2 continham a análise da mobilidade torácica, sendo um estudo transversal e outro de caso controle. Ainda são escassos na literatura estudos de intervenção que avaliem os parâmetros de função respiratória, entre eles a cirtometria.

Os valores de normalidade para o coeficiente de amplitude e a diferença entre a inspiração e a expiração máximas são citados na literatura, variando de 6 a $7 \mathrm{~cm}$, e de 5 a $11 \mathrm{~cm}$, sendo que medidas de 3 a $4 \mathrm{~cm}$ já estariam relacionadas a uma perda de $20 \%$ da capacidade pulmonar $^{21,22}$. Nos resultados demonstrados no Quadro 2 , os valores variam de 3 a $8 \mathrm{~cm}$ em média, considerando o desvio padrão.

$\mathrm{Na}$ amostra estudada, ficou evidenciado que os indivíduos com DP, antes de serem submetidos às intervenções e mesmo depois delas, apresentam um tórax rígido, ou seja, com pouca mobilidade, considerando as medições em nível axilar e xifoidiano; já a mobilidade abdominal parece ter sido influenciada pelas intervenções. Isto pode ser explicado pelo fato de esses indivíduos, quando submetidos a um exercício físico que demanda incremento da função pulmonar e consequentemente da movimentação da caixa torácica por tê-la rígida, acabam por requisitar a mobilidade abdominal que, supostamente, se traduz em uma maior atuação do músculo diafragma, "compensando" essa mobilidade reduzida do tórax para atender a demanda.

No GF que realizou exercícios ditos funcionais, assemelhados às tarefas da vida diária e que apresentam maior variabilidade de movimentos, posturas e mobilidade, esse incremento na mobilidade abdominal foi maior na casa de $1,0 \mathrm{~cm}$. Não existe ainda na literatura um valor de diferença mínima clinicamente importante para cirtometria, aliada à escassez de estudos de intervenção de Fisioterapia na DP que avaliem esse parâmetro, assim este resultado, no momento, não pode ser comparado.

Neste estudo, podem se considerar como limitação o fato de os participantes não terem realizado nenhum tipo de intervenção de Fisioterapia respiratória, portanto, os achados foram produzidos de forma "indireta" como consequência dos exercícios funcionais, da bicicleta e do exergame. Talvez se aliados a essas intervenções tivessem sido realizados exercícios específicos para ganho de mobilidade, os resultados seriam diferentes dos encontrados; outro fator a ser considerado é o tamanho da amostra relativamente reduzida, o que não possibilita realizar inferências para essa população com DP.

\section{CONCLUSÃO}

Neste estudo, foram comparados os valores da cirtometria ou mobilidade toracoabdominal de idosos com DP, antes e depois de serem submetidos a três diferentes intervenções (treino funcional, bicicleta estacionária e exergame): não foram encontradas diferenças nos níveis axilar e xifoide; apenas no nível umbilical encontrou-se um aumento maior no grupo submetido ao treino funcional.

Os resultados encontrados neste estudo sugerem que a população de idosos com DP, quando submetida ao tratamento fisioterapêutico, além da funcionalidade motora, deva também ter enfatizada sua funcionalidade respiratória/pulmonar, já em caráter preventivo, ou seja, antes que essa disfunção se manifeste, acarretando graves consequências, inclusive o óbito.

\section{REFERÊNCIAS}

1. FERRAZ, D. D.; TRIPPO, K. V.; DUARTE, G. P. et al. The effects of functional training, bicycle exercise, and exergaming on walking capacity of elderly patients with Parkinson disease: A pilot randomized controlled single-blinded trial. Arch. phys. med. rehabil., Chicago, v. 99, n. 5, p. 826-833, 2018.

2. GUIMARÃES, D. et al. Using the spirometry to indicate respiratory exercises for elderly with Parkinson's disease. Fisioter. mov., Curitiba, v. 31, n. 0, 2018. Disponível em: http://www.scielo.br/scielo. php?script=sci_arttext $\&$ pid=S0103-51502018000100218\&lng=en\&tln g=en. Acesso em: 15 ago. 2019.

3. SILVEIRA, R. A. et al. The effects of functional training and stationary cycling on respiratory function of elderly with Parkinson disease: a pilot study. Fisioter. mov., Curitiba, v. 31, n. 0, 2018. Disponível em: http://www.scielo.br/scielo.php?script=sci_arttext\&pid=S0103$-51502018000100215 \&$ Ing=en\&tIng=en. Acesso em: 15 ago. 2019.

4. RAMOS, M. L. et al. Análise de parâmetros pneumofuncionais em pacientes com doença de Parkinson: estudo piloto. Rev. Bras. Neurol., Rio de Janeiro, v. 50, p. 38-43, 2014.

5. FERREIRA, F. V.; CIELO, C. A.; TREVISAN, M. E. Aspectos respiratórios, posturais e vocais da doença de Parkinson: considerações teóricas. Rev. CEFAC, São Paulo, v. 13, n. 3, p. 534-540, 2010.

6. VIEIRA F. D. M. Plataforma de apoio à terapia de reabilitação e manutenção de doentes de Parkinson. 2013. Dissertação (Mestrado em Engenharia) - Universidade do Porto, Porto, Portugal, 2013.

7. TOMLINSON, C. L. et al. Physiotherapy for Parkinson's disease: a comparison of techniques. Cochrane database syst. rev., Oxford, v. 17, n. 6, p. 121, June 2014 .

8. GIL-GÓMEZ, J. A. et al. Effectiveness of a Wii balance board-based system (eBaViR) for balance rehabilitation: a pilot randomized clinical trial in patients with acquired brain injury. J. neuroengineering rehabil., 
Análise da mobilidade toracoabdominal de idosos com doença de parkinson, submetidos ao treinamento funcional, bicicleta estacionária e exergame: um ensaio clínico randomizado

London, v. 8, p. 30, May 2011. DOI: 10.1186/1743-0003-8-30.

9. CALDEIRA, V. da S. et al. Precisão e acurácia da cirtometria em adultos saudáveis. J. Bras. Pneumol., Brasília, v. 33, n. 5, p. 519-526, 2007

10. HOEHN, M. M. Parkinsonism: onset, progression, and mortality. p. Neurology, New York, v.17, n. 5, p. 427-442, May 1967.

11. CISTERNAS, N. S. ACSM guidelines for exercise testing and prescription. 10th. ed. 2018. Disponível em: https://www.academia.edu/36843773/ACSM_Guidelines_for_Exercise_Testing_and Prescription_10th. Acesso em: 29 ago. 2019.

12. KEUS, S. et al. European physiotherapy guideline for Parkinson's deseases: information for clinicians. [S.I.]: ParkinsonNet, 2014. p. 12

13. WILSON, R. C.; JONES, P. W. A comparison of the visual analogue scale and modified Borg scale for the measurement of dyspnoea during exercise. Clin. sci., Oxford, v. 76, n. 3, p. 277-282, 1989.

14. CANNING, C. G. et al. Exercise for falls prevention in Parkinson disease. Neurology, New York, v. 84, n. 3, p. 304-312, 2015.

15. KARVONEN, M. J.; KENTALA, E.; MUSTALA, O. The effects of training on heart rate; a longitudinal study. Ann. med. exp. biol. fen., Helsinki, v. 35 , n. 3 , p. $307-315,1957$

16. ALVES DA ROCHA, P.; MCCLELLAND, J.; MORRIS, M. E. Complemen- tary physical therapies for movement disorders in Parkinson's disease: a systematic review. Eur. j. phys. rehabil. med., Torino, v. 51, n. 6, p. 693-704, 2015.

17. ABBRUZZESE, G. et al. Rehabilitation for Parkinson's disease: current outlook and future challenges. Parkinsonism Relat. Disord., Kidlington, v. 22 , supl 1, p. S60-64, 2016.

18. FERREIRA, J. C.; PATINO, C. M. What does the $p$ value really mean? J. bras. pneumol., Brasília, v. 41, n. 5, p. 485-485, 2015.

19. CARDOSO, S. R.X.; PEREIRA, J. S. Análise da função respiratória na doença de Parkinson. Arquivos neuropsiquiatr., São Paulo v. 60, n. 1, p. 91-95, 2002.

20. TASCA, C.; SCHUSTER, R. C.; ALVARENGA, L.F. C. Força muscular respiratória e mobilidade torácica em portadores de doença de Parkinson. Revista de Atenção à Saúde, São Paulo, v. 12, n. 42, 2014. Disponível em: http://seer.uscs.edu.br/index.php/revista_ciencias_saude/article/ view/2275. Acesso em: 20 ago. 2019.

21. COSTA, H. da C. et al. Alterações funcionais respiratórias na doença de Parkinson. [S.I.: s.n.], 2016.

22. LANZA, F. de C. et al. Chest wall mobility is related to respiratory muscle strength and lung volumes in healthy subjects. Respir. care, Dallas, v. 58, n. 12, p. 2107-2112, 2013.

Submetido em: 04/11/2019

Aceito em: 30/11/2019 